\title{
El uso de recursos electrónicos en las bibliotecas universitarias del área científica en la Universidad Nacional Autónoma de México
}

\author{
The use of electronic resources in the university libraries of the scientific area \\ of the Universidad Nacional Autónoma de Méjico
}

\author{
María del Carmen Negrete Gutiérrez \\ Centro Universitario de Investigaciones Bibliotecológicas, Universidad Nacional Autónoma de México
}

\begin{abstract}
Resumen
El desarrollo de colecciones en las bibliotecas universitarias se ha convertido en una actividad de creciente importancia, puesto que es una actividad que esta en medio y es determinante de las otras actividades bibliotecarias. Este hecho hace que el estudio del uso de las colecciones y los recursos, por parte de las comunidades universitarias, revista una importancia especial. En este estudio, nos enfocamos en la comunidad científica de la Universidad Nacional Autónoma de México (UNAM) y pretendemos indagar sobre las variables relacionadas con la forma en que estas comunidades hacen uso de las colecciones y los recursos electrónicos de sus bibliotecas, ya que consideramos que estos resultados pueden arrojar conclusiones interesantes para las políticas de desarrollo de colecciones y para la planeación y selección de recursos. La encuesta se aplicó en 23 bibliotecas universitarias del área científica de la UNAM.
\end{abstract}

Palabras clave: Bibliotecas universitarias. Recursos digitales. Universidad Nacional Autónoma de México.

\section{Introducción}

Generalmente se afirma que, "Las necesidades de información son una de las partes centrales en la teoría de la bibliotecología y de la documentación, ya que las actividades bibliotecarias y de información se sustentan en satisfacer las necesidades y demandas de nuestros usuarios" (Calva, 1998, p. 47). Lo señalado se sustenta en la afirmación de Rendón, quien establece que, "en la relación información-usuario-documentoinstitución informativa, las necesidades de información del usuario se relacionan con la actividad bibliotecaria correspondiente al desarrollo de colecciones" (Rendón, 1997, p. 123).

Sin duda, las dos aseveraciones confirman que, el desarrollo de colecciones constituye una de las funciones más importantes en la biblioteca

\begin{abstract}
Collection development in University libraries has increasingly become an activity of utmost importance as it is determinant of other library functions. Thus, a user analysis of the collection and resources by the university community is highly important. The study seeks to inquire into the variables directly related to the user behavior of the scientific community of the National Autonomous University of Mexico (UNAM) regarding their library collections and electronic resources. We believe that the conclusions my be important for collection development policies and planning and resources selection. The survey was conducted at 23 UNAM science university libraries.
\end{abstract}

Keywords: University libraries. Electronic resources. Collections use. Universidad Nacional Autónoma de México.

universitaria o centro de documentación, tiene como objetivos los de (Negrete, 2006, p. 141):

formar y orientar una colección de recursos informativos que se ajusten al objetivo de la institución de la que depende, y a las necesidades y demandas de información de la comunidad, manteniendo un adecuado balance cualitativo y cuantitativo entre las diferentes áreas de interés y entre los diversos tipos de recursos representados en distintos formatos.

Ante esta afirmación, los responsables del desarrollo de colecciones tienen que estudiar diversos aspectos relacionados con los usuarios, lo que permitirá formar y mantener colecciones coherentes y adecuadas. Es necesario, por tanto, identificar quiénes son nuestros usuarios, cuáles son sus necesidades de información, el nivel de esas necesidades, cómo realizan la 
búsqueda de información, qué tipo de recursos utilizan, cómo y dónde los utilizan y, finalmente, saber si hay o no una satisfacción de necesidades informativas.

En el caso concreto de las comunidades académicas universitarias, las necesidades de información se encuentran ligadas, principalmente, con las funciones sustantivas de la universidad, entre las que se encuentran: la investigación, la docencia y el aprendizaje, la difusión y la extensión de la cultura dentro y fuera del ámbito universitario.

Sin embargo, para cumplir con las funciones señaladas se requiere que el profesor, investigador y alumno estén mejor informados, es decir, que adquieran mayor y mejor infraestructura de conocimientos, ya que la información es una factor importante para el desarrollo científico, humanístico y tecnológico, y para las mejoras sociales, educativas y culturales.

En la actualidad, las bibliotecas universitarias mexicanas, principalmente las públicas, enfrentan el crecimiento de la oferta de la información con costos crecientes que afectan, en ocasiones, los limitados presupuestos con que cuentan para realizar sus adquisiciones. A esto hay que aunar el propio auge de los diversos medios electrónicos que, si bien permiten una mayor cobertura, diseminación, acceso y transferencia de la información, también representan, en buena medida, costos mayores para su adquisición o suscripción.

En este contexto, el papel que juega la biblioteca universitaria o el centro de documentación es de suma importancia pues debe ser un sistema eficiente y dinámico que ofrezca conocimientos y datos que apoyen las necesidades de información de su comunidad.

Satish mencionaba en 1994 que, en el contexto de otros países, aun de entre las diversas áreas (humanidades, ciencias sociales y ciencias duras) es muy poco lo que conocemos acerca de las necesidades de información. Asimismo, son pocos los estudios que han sido desarrollados sobre las necesidades de información de los académicos, y no precisamente de académicos de las ciencias sociales (Satish, 1994, p. 30). Por otro lado, Abad García apuntaba en 2005 (p. 17) que,

si la realidad estuviera gobernada por la lógica, la línea de investigación más desarrollada en el contexto de la biblioteconomía y documentación sería la del estudio de las necesidades de información de los usuarios, habida cuenta de que los sistemas y servicios de información existen en virtud de su satisfacción.
Sin duda, lo señalado por los autores no deja de ser verdad. En el caso concreto de México son escasos los estudios realizados sobre las necesidades de información o sobre el uso que hacen las comunidades universitarias de los diversos recursos.

Sin lugar a dudas, los integrantes de las comunidades académicas tienen necesidades de información diferentes y se debe considerar que el surgimiento y manifestación de éstas pueden estar influidas por diversos factores, tales como (Calva, 2004, p. 50):

- Lugar donde realizan su investigación o trabajo académico.

- Los objetivos, funciones y políticas de la institución donde laboran los académicos.

- Las áreas de actividad académica.

- Los temas de investigación.

- Los tipos de actividad o de investigación.

- Los apoyos para realizar labores.

- Los conocimientos o experiencia en el tema o área de su competencia.

- La experiencia en la utilización de métodos de investigación.

- El uso de recursos y fuentes informativas, así como el acceso a las diversas unidades de información.

Pero no se debe perder de vista que las necesidades de información son cambiantes a lo largo del tiempo y, por lo tanto, esos perfiles deben ser actualizados y revisados a la luz de nuevas necesidades o demandas informativas. Sin perder de vista que en algunos casos, las necesidades de los usuarios podrían estar influenciadas por las innovaciones tecnológicas de la información, las telecomunicaciones, la industria de la información y muchas veces por la propaganda comercial en todos los medios. Todo lo anterior genera mayor demanda de modernos servicios y por ende de recursos informativos.

Calva González (2004, p. 25), señala que "el usuario en muchas ocasiones no es capaz de expresar lo que necesita pues a veces los usuarios no saben en forma precisa lo que quieren o necesitan" y es el bibliotecólogo o documentalista quienes hacen que el usuario verbalice sus necesidades o demandas de información de una forma clara, para relacionarlas con la información adecuada que se localiza en sus bibliotecas.

Ante estas aseveraciones, deberíamos atender lo señalado por González Teruel (2005, p. 23), quien apunta que

el estudio de los usuarios de la información constituye un conjunto de investigaciones cuyos resultados permitan planificar y mejorar los sistemas de información. En general, la observación sistemática del usuario ofrece una herramienta de gran valor para tomar decisiones tanto desde el punto de 
vista de la gestión de las unidades de información como desde la perspectiva del bibliotecario o documentalista que día a día atiende sus peticiones.

En lo señalado por la autora, se infiere que la observación sistemática del usuario en el uso que hace de las colecciones impresas y electrónicas permitiría obtener indicadores para planificar el desarrollo de colecciones en la biblioteca universitaria. Aunado a esto, es necesario conocer el entorno de las colecciones y recursos que se localizan en las bibliotecas, pues el entorno de alguna manera puede influir en el posible impacto que causan en ellos los actuales recursos electrónicos frente a los recursos tradicionales o convencionales que se localizan en estas instituciones.

Con estos antecedentes se decidió llevar a cabo un estudio de campo basado en entrevistas personales a personas idóneas que tuvieran conocimiento sobre la aceptación y el uso que hacen sus usuarios de los recursos impresos y electrónicos en Bibliotecas Universitarias del área científica de la UNAM.

Los objetivos del estudio son el obtener indicadores sobre la aceptación y el uso que hacen sus usuarios de los recursos electrónicos frente a los recursos impresos; y, así también, conocer el entorno de las colecciones impresas y electrónicas.

Para cumplir con el objetivo señalado, se decidió como método de interrogación la consulta mediante el cuestionario.

Todas las preguntas fueron libres en el sentido de que el responsable de la biblioteca interrogado no estaba obligado a responder, pudiendo siempre negarse a hacerlo o declararse sin opinión. La preparación del cuestionario se conformó con preguntas abiertas y cerradas.

El estudio de campo se realizó durante el primer semestre de 2006, periodo en el que se llevó a cabo la entrevista a los jefes o coordinadores de 29 bibliotecas universitarias del área científica de la UNAM, considerando que ellos tienen conocimiento y acceso a las estadísticas de uso que se recopilan mensualmente.

\section{Metodología}

Primeramente, se definieron los puntos de interés para el estudio y luego se diseñó un cuestionario, cuidando que las preguntas fueran claras y cubrieran diferentes aspectos, los cuales quedaron agrupados en cinco grandes rubros: profesión de los jefes o coordinadores de las bibliotecas universitarias, recursos electrónicos que utilizan los usuarios, recursos tradicionales que utilizan los usuarios, idioma de prefe- rencia en la información, e infraestructura con la que cuenta la biblioteca para ofrecer recursos electrónicos.

Se procedió a definir la muestra de bibliotecas universitarias que habrían de estudiarse. Para definir la muestra se consultó el "Directorio del Sistema Bibliotecario 2004" (Directorio..., 2004). De éste, se obtuvo que en la UNAM el Subsistema de Licenciatura y Posgrado esta conformado por un total de 50 bibliotecas universitarias. De este total, 29 son del área de científica.

Con base en estos resultados, se procedió a realizar los contactos personales para explicar el objetivo del estudio y solicitar la cooperación de los jefes o coordinadores de las 29 bibliotecas universitarias. De ese total dieron respuesta de 24 bibliotecas.

Dichos resultados se presentan a continuación cuantificados a través de cuadros, acompañados de sus correspondientes análisis.

\begin{tabular}{ll}
\hline Maestría en Bibliotecología & 3 \\
Lic. en Bibliotecología & 3 \\
Técnico en Biblioteconomía & 1 \\
Lic. en Medicina veterinaria y zootecnia & 3 \\
Lic. en Historia & 1 \\
Lic. en Ingeniería mecánica y eléctrica & 1 \\
Lic. en Medicina & 1 \\
Lic. en Derecho & 1 \\
Especialidad en Medicina & 1 \\
Mtro. en Administración de información & 1 \\
Ingeniero en computación & 1 \\
Pasante de la Lic. en Contaduría pública & 1 \\
Pasante de la Lic. en Pedagogía & 1 \\
Estudios medio superior & 1 \\
Lic. en Matemáticas & 1 \\
Lic. en Administración de empresas & 1 \\
Lic. en Contabilidad & 1 \\
No contestó & 1 \\
\hline
\end{tabular}

Cuadro 1. Estudios profesionales de los responsables de las bibliotecas

Es preocupante que siendo la UNAM sede de uno de los programas más antiguos en Bibliotecología en América Latina, las jefaturas o coordinaciones de las bibliotecas universitarias de la propia institución sean todavía ocupadas en un porcentaje importante del casi $67 \%$ de personal formado en otras áreas, aunque es satisfactorio observar que casi todos los que contestaron el cuestionario tienen estudios de licenciatura y maestría. Solo en un caso no obtuvimos respuesta, probablemente porque no cuenta con estudios profesionales terminados. 


\begin{tabular}{|l|c|c|}
\cline { 2 - 3 } \multicolumn{1}{c|}{} & $\begin{array}{c}\text { Mayor } \\
\text { frecuencia }\end{array}$ & $\begin{array}{c}\text { Menor } \\
\text { frecuencia }\end{array}$ \\
\hline Libros o monografías & 23 & 1 \\
\hline Obras de consulta & 11 & 13 \\
\hline $\begin{array}{l}\text { Publicaciones } \\
\text { periódicas }\end{array}$ & 19 & 3 \\
\hline Documentos técnicos & 5 & 4 \\
\hline Periódicos & 1 & 6 \\
\hline Micropelículas & - & 7 \\
\hline Audiovisuales & 3 & 7 \\
\hline Mapas & - & 7 \\
\hline Tesis & 10 & 13 \\
\hline Folletos & 1 & - \\
\hline Notas de cursos & 1 & - \\
\hline CD's de libros & 1 & 1 \\
\hline Prácticas escolares & 1 & - \\
\hline Diapósitivas & 1 & - \\
\hline
\end{tabular}

Cuadro 2. Recursos tradicionales que utiliza la comunidad

En opinión de los encuestados, las fuentes informativas como los libros, las publicaciones periódicas, las obras de consulta y las tesis, son los recursos tradicionales o convencionales utilizados con mayor frecuencia por los usuarios, lo cual no resulta ninguna sorpresa, dado que el Subsistema de Licenciatura y posgrado contaba en 2005 con una población de alumnos de cerca de 200.000. Por tanto, los alumnos son en su mayoría los usuarios en las bibliotecas. Estos datos podrían posiblemente orientar las prioridades de digitalización en la Dirección General de Bibliotecas de la UNAM.

La menor frecuencia en el uso de algunos de los recursos que utilizan los usuarios podría deberse a la existencia de los mismos en formato electrónico. Es claro que el uso de los servicios tradicionales continúa. Es el caso de la sala de lectura, en el $79 \%$ (19) de las bibliotecas; consulta en el $75 \%$ (18) y el préstamo en sala, a domicilio e interbibliotecario en casi el $71 \%$ (17) de las bibliotecas. Esto podría justificarse debido a que los profesores, investigadores y estudiantes con frecuencia demandan materiales antiguos o necesitan consultar cualquiera de los volúmenes de una colección. Por lo que las publicaciones electrónicas no siempre responden a sus necesidades informativas. Algunos de los servicios tan utilizados en otros años como la elaboración de bibliografías y las búsquedas especializadas, ahora con la presencia de recursos electrónicos se usan menos.

Es claro que la comunidad en este tipo de bibliotecas universitarias han aceptado los recursos electrónicos. Esto sucede en el casi $79 \%$ (19) de las bibliotecas. El alto uso de los recursos electrónicos por parte de este colectivo se debe a que son ellos los que están más actualizados en el uso y manejo de recursos electrónicos y probablemente cuentan en sus hogares con equipos informáticos, lo que les ha permitido el desarrollo de habilidades informativas.

\begin{tabular}{|l|c|c|c|}
\cline { 2 - 4 } \multicolumn{1}{c|}{} & $\begin{array}{c}\text { Continua } \\
\text { su uso }\end{array}$ & $\begin{array}{c}\text { Se usan } \\
\text { más }\end{array}$ & $\begin{array}{c}\text { Se usan } \\
\text { menos }\end{array}$ \\
\hline Sala de lectura & 19 & 2 & 1 \\
\hline Consulta & 18 & 2 & 2 \\
\hline Préstamo en sala & 17 & 5 & 1 \\
\hline $\begin{array}{l}\text { Préstamo } \\
\text { a domicilio }\end{array}$ & 16 & 3 & - \\
\hline $\begin{array}{l}\text { Préstamo } \\
\text { interbibliotecario }\end{array}$ & 12 & 6 & 2 \\
\hline $\begin{array}{l}\text { Orientación } \\
\text { a usuarios }\end{array}$ & 11 & 8 & 2 \\
\hline $\begin{array}{l}\text { Búsquedas } \\
\text { especializadas }\end{array}$ & 10 & 6 & 3 \\
\hline $\begin{array}{l}\text { Elaboración } \\
\text { de bibliografías }\end{array}$ & 2 & 3 & 5 \\
\hline Reserva & 5 & 2 & 2 \\
\hline Fotocopiado & 16 & 3 & 1 \\
\hline
\end{tabular}

Cuadro 3. Uso de los servicios tradicionales

\begin{tabular}{|l|c|c|}
\cline { 2 - 3 } \multicolumn{1}{c|}{} & $\begin{array}{c}\text { Los han } \\
\text { aceptado }\end{array}$ & $\begin{array}{c}\text { No los han } \\
\text { aceptado }\end{array}$ \\
\hline $\begin{array}{l}\text { Estudiantes } \\
\text { de licenciatura }\end{array}$ & 18 & 2 \\
\hline Estudiantes de posgrado & 18 & - \\
\hline $\begin{array}{l}\text { Profesores } \\
\text { de tiempo completo }\end{array}$ & 18 & 3 \\
\hline Profesores/investigadores & 18 & 2 \\
\hline Profesores de asignatura & 19 & 3 \\
\hline Técnicos académicos & 18 & 3 \\
\hline Usuarios externos & 15 & 2 \\
\hline Tesistas & 17 & 1 \\
\hline
\end{tabular}

Cuadro 4. Aceptación de recursos electrónicos por la comunidad

En el caso de los profesores e investigadores, se observa que todavía en algunas bibliotecas no los han aceptado. Esto puede ser consecuencia de la edad que varios de ellos tienen o quizá porque son docentes tradicionales y no aceptan el cambio.

La disparidad de los resultados reflejados en el Cuadro 5 podría deberse a que el acceso a los recursos electrónicos a través de las bibliotecas es limitado, ya que éstas tienen poco equipo disponible para consultar recursos electrónicos. 
Habría que tomar en cuenta que los investigadores y profesores de tiempo completo cuentan con equipo en sus cubículos, desde los que pueden acceder a la información electrónica, medios de los que los estudiantes no disponen.

\begin{tabular}{|c|c|c|}
\hline & Frecuente & Ocasional \\
\hline Catálogo en línea & 17 & - \\
\hline \multicolumn{3}{|c|}{ Bases de datos en línea: } \\
\hline $\begin{array}{l}\text { a. A través del portal de la } \\
D G B\end{array}$ & 18 & 1 \\
\hline $\begin{array}{l}\text { b. Contratadas } \\
\text { directamente por la } \\
\text { dependencia }\end{array}$ & 3 & 2 \\
\hline c. Gratuitas & 6 & 2 \\
\hline d. Creadas por la Biblioteca & 6 & 3 \\
\hline $\begin{array}{l}\text { Bases de datos en CD } \\
\text { ROM }\end{array}$ & 4 & 3 \\
\hline \multicolumn{3}{|c|}{ Revistas electrónicas de texto completo: } \\
\hline $\begin{array}{l}\text { a. A través del sitio de la } \\
D G B\end{array}$ & 16 & 2 \\
\hline $\begin{array}{l}\text { b. Contratadas } \\
\text { directamente por la } \\
\text { dependencia }\end{array}$ & 2 & 1 \\
\hline Obras de consulta en línea & 2 & 6 \\
\hline $\begin{array}{l}\text { Obras de consulta en CD- } \\
\text { ROM }\end{array}$ & 4 & 2 \\
\hline $\begin{array}{l}\text { Acceso a sitios Web de la } \\
\text { especialidad }\end{array}$ & 8 & 6 \\
\hline $\begin{array}{l}\text { Diseminación selectiva de } \\
\text { información }\end{array}$ & 2 & 3 \\
\hline Libros electrónicos & 4 & 5 \\
\hline Videos digitales & 1 & 1 \\
\hline Documentos técnicos & - & 2 \\
\hline Tesis digitales & 2 & 5 \\
\hline Periódicos electrónicos & 2 & - \\
\hline Mapas electrónicos & - & 1 \\
\hline
\end{tabular}

Cuadro 5. Servicios electrónicos usados por su comunidad

Esta disparidad podría deberse también a que el $54 \%(13)$ de las bibliotecas universitarias cuentan con un departamento de cómputo independiente de la biblioteca, en el cual ofrecen el servicio de acceso a información electrónica a los usuarios y las estadísticas de uso no están controladas por la biblioteca. Pero es claro que los responsables de las bibliotecas tendrían que estar informándose del uso que hace su comunidad de recursos electrónicos. El libro electrónico en la Universidad se usa más como un mecanismo de consulta, que como uno de lectura, es más un recurso para la consulta de información técnica que literaria; esto podría justificar el bajo uso de libros electrónicos.

\begin{tabular}{|c|c|c|c|c|}
\hline & 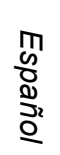 & 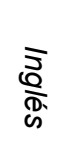 & 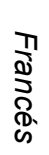 & \\
\hline Estudiantes de licenciatura & 23 & 6 & - & - \\
\hline Estudiantes de posgrado & 12 & 19 & 2 & 1 \\
\hline $\begin{array}{l}\text { Profesores de tiempo } \\
\text { completo }\end{array}$ & 10 & 17 & 2 & 1 \\
\hline Profesor/investigadores & 9 & 20 & 2 & - \\
\hline Profesores de asignatura & 13 & 17 & 2 & - \\
\hline Técnicos académicos & 14 & 14 & 1 & - \\
\hline Usuarios externos & 20 & 7 & 1 & 1 \\
\hline Tesistas & 18 & 9 & - & - \\
\hline
\end{tabular}

Cuadro 6. Idioma de preferencia de la información

Cómo cabía esperar, el idioma español es el preferido por los estudiantes para utilizar la información, lo cual contrasta con que la mayor parte de los recursos electrónicos están en inglés. Sería interesante conocer la satisfacción de las necesidades de información con recursos en inglés. Se destaca que los usuarios están interesados o preocupados por aprender otros idiomas.

\begin{tabular}{|l|c|}
\hline Nivel de entrenamiento & Grado \\
\hline Muy general & 7 \\
\hline Bien & 3 \\
\hline Exhaustivo & 4 \\
\hline No recibieron & 6 \\
\hline No contestaron & 4 \\
\hline
\end{tabular}

Cuadro 7. Nivel de entrenamiento del personal de la biblioteca

Los resultados indican la necesidad de reforzar el entrenamiento del personal para hacerlo mas especializado.

Paralelamente, un problema que se ha observado al analizar la formación que reciben los profesionales que trabajan en las bibliotecas de la UNAM, es que su Dirección General de Bibliotecas imparte a través de sus técnicos académicos (no necesariamente capacitados en todos los casos), cursos de formación dirigidos a los responsables de todas las bibliotecas del Sistema, pero no a los responsables de los servicios, a pesar de que son ellos quienes interactúan con los usuarios. La DBG tiene establecido en su Reglamento General, que una de sus funciones es aplicar el plan de capacitación, formación y desarrollo profesional del personal que labora en las bibliotecas. 


\begin{tabular}{|c|c|c|c|c|}
\hline & 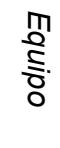 & 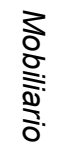 & 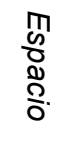 & $\begin{array}{l}20 \\
\mathbb{Q}\end{array}$ \\
\hline Suficiente & 4 & 7 & 9 & 11 \\
\hline Insuficiente & 15 & 12 & 10 & 9 \\
\hline Buen estado & 2 & 3 & 3 & 3 \\
\hline Mal estado & 1 & - & 1 & - \\
\hline Insuficiente en buen estado & 1 & 1 & 1 & 1 \\
\hline No contestó & 1 & 1 & 1 & 1 \\
\hline
\end{tabular}

Cuadro 8. Infraestructura para ofrecer recursos electrónicos

A la vista de los datos expuestos, se constata que existe un claro déficit en lo que a infraestructura para ofrecer servicios electrónicos se refiere. Respecto al equipo, éste es insuficiente en el $62 \%$ (15) de las bibliotecas; en cuanto a mobiliario, en el $50 \%$ (12) de las bibliotecas y en espacio en el $41 \%$ (10) de ellas.

Respecto a las causas de estas deficiencias, quizá en parte estén relacionadas con la alta demanda que en este momento llevan a cabo los estudiantes. Por otra parte, se confirma de nuevo lo que ya se había señalado anteriormente y es que hay poco equipo disponible en las bibliotecas en contraste con la infraestructura disponible en cubículos para profesores e investigadores.

\begin{tabular}{|l|l|}
\hline $\begin{array}{l}\text { Actualizar los equipos de cómputo } \\
\text { para el área de servicios }\end{array}$ & 8 \\
\hline Ampliación de los espacios & 4 \\
\hline Red con mayor capacidad & 1 \\
\hline Obtención de recursos informativos & 1 \\
\hline Obtención de recursos humanos & 1 \\
\hline Presupuesto para adquirir equipo & 5 \\
\hline Mejorar mobiliario & 3 \\
\hline Ampliación de instalaciones & 3 \\
\hline
\end{tabular}

Cuadro 9. Sugerencias personales sobre la infraestructura para ofrecer servicios

Sin duda la sugerencia principal es la necesidad de actualizar los equipos de cómputo para el área de servicios, lo cual es comprensible si tenemos en cuenta la rapidez con que aparecen nuevos formatos electrónicos. Esta necesidad es objeto de preocupación en el $33 \%$ (8) de las bibliotecas.

\section{A manera de conclusión}

En primer lugar, debemos tener en cuenta que la comunicación académica no es igual en todas las disciplinas, debido a la naturaleza del tema de estudio o de investigación; $y$, por tanto, como es tratado en las aulas y en los cubículos de los investigadores, determina la forma de acceso o disponibilidad de la información.

Otra observación importante y que no se puede soslayar, es la edad de algunos profesores/investigadores, la cual constituye un importante obstáculo para el aprendizaje de nuevas tecnologías y, por lo tanto, para el uso de los recursos electrónicos. La dificultad de adaptación al nuevo escenario está además condicionada porque la mayoría de ellos tienen una formación que podríamos llamar de tipo "tradicional".

Las dificultades de implantación de los formatos electrónicos están muy relacionadas con su reciente aparición. En México, según los datos obtenidos de la DGB, la mayor parte de las publicaciones electrónicas en texto completo están fechadas a partir de 1995.

\section{Referencias}

Calva Gonzáles, Juan José. La investigación sobre las necesidades de información en comunidades de usuarios. Vol. 18 no.37 (julio-diciembre 2004) 25

Calva González, Juan José. Las necesidades de información de la comunidad académica como base en el desarrollo de colecciones. // Primer seminario internacional sobre desarrollo de colecciones. México: UNAM.CUIB, 1998. p. 47-56

Abad, García. Presentación. // Los estudios de necesidades y usos de la información: fundamentos y perspectivas actuales. España: TREA, 2005. 181p.

Negrete Gutiérrez, María del Carmen. El impacto del entorno electrónico en la planificación del desarrollo de colecciones: las bibliotecas universitarias del área de humanidades de la UNAM. Madrid: Negrete Gutiérrez, 2006. 502 p. (Tesis de doctorado en Ciencias de la Información. Universidad Complutense de Madrid. Facultad de Ciencias de la Información. Departamento de Biblioteconomía y Documentación)

Rendón Rojas, Miguel Angel. Bases teóricas y filosóficas de la bibliotecología. México: UNAM. Centro Universitario de Investigaciones Bibliotecológicas, 1997. 148p.

Satish, N.G. Attitud toward information: a study of social scientists. New Delhi: Concept Publishing, 1994.

Aurora González Teruel. Los estudios de necesidades y usos de la información: fundamentos y perspectivas actuales. España: TREA, 2005. 181p. 